\title{
A Comparison of the Predictive Performance of Different Methods of Kidney Function Estimation in a Well-Characterized HIV-Infected Population
}

\author{
Katherine Barraclough ${ }^{\mathrm{a}}$ Lee $\mathrm{Er}^{\mathrm{a}} \quad$ Francisco $\mathrm{Ng}^{\mathrm{b}} \quad$ Marianne Harris ${ }^{\mathrm{b}}$ \\ Julio Montaner $^{\mathrm{b}}$ Adeera Levin ${ }^{\mathrm{a}}$ \\ a Division of Nephrology, University of British Columbia, and ' ${ }^{\mathrm{b}}$ AIDS Research Program, St. Paul's Hospital, \\ Vancouver, B.C., Canada
}

\section{Key Words}

Creatinine $\cdot$ Creatinine clearance $\cdot$ Cystatin $\cdot$ Estimation

equations $\cdot$ Glomerular filtration rate $\cdot$ Human

immunodeficiency virus

\begin{abstract}
Background: Glomerular filtration rate (GFR) estimation equations have never been validated in the HIV population. This pilot study aimed to compare all currently available methods of kidney function assessment with nuclear GFR in HIV-infected adults. Methods: Patients underwent GFR measurement with $99 \mathrm{~m}$ Tc-diethylenetriaminepentaacetic acid (Tc-99m Pentetate), and measured values were compared with results of creatinine-based estimation equations [abbreviated 4-variable Modification of Diet in Renal Disease (MDRD) formula and Cockcroft-Gault (CG) formulae], 24hour urine creatinine clearance and estimated cystatin $C$ GFR. Results: Twenty-seven HIV-infected adults were studied. Most were male and Caucasian, with a mean age of 52 years. Median CD4 was 290 cells $/ \mathrm{mm}^{3}, 70 \%$ of patients had HIV RNA $<50$ copies/ml and all were receiving highly active antiretroviral therapy (median 5 drugs). Median Tc-99m Pentetate-GFR was $91 \mathrm{ml} / \mathrm{min} / 1.73 \mathrm{~m}^{2}$. Despite greater bias and similar accuracy, the MDRD formula was more precise than the CG formula, regardless of whether CG estimations were corrected for ideal body weight or body surface area. Relative accuracy within $30 \%$ of nuclear GFR was greater for the
\end{abstract}

MDRD formula than for all other methods. The performance of 24-hour urine creatinine clearance was similar to that of the MDRD formula for patients with GFR $<90 \mathrm{ml} / \mathrm{min} / 1.73$ $\mathrm{m}^{2}$, although it performed less well at higher GFR. The performance of cystatin C GFR was inferior to that of all the creatinine-based methods. Conclusions: While no method of kidney function estimation performed highly, both 24-hour urine creatinine clearance and the MDRD formula performed with a level of precision and accuracy sufficient for clinical decision making. Our findings support the preferential use of the MDRD formula in the treated HIV population and suggest that there are no HIV-specific factors that limit equation applicability. Larger validation studies are needed to confirm our findings and allow generalization to the HIV population at large.

Copyright $\odot 2008$ S. Karger AG, Basel

\section{Introduction}

The accurate assessment of kidney function in the $\mathrm{HIV}$-infected population is of paramount importance. HIV treatment with highly active antiretroviral therapy (HAART) involves complex drug regimens that are often accompanied by significant side effects and potential drug interactions. A significant number of antiretroviral drugs undergo renal elimination and are dosed according to kidney function [1]. The HIV-infected population

\section{KARGER}

Fax +41613061234 E-Mail karger@karger.ch www.karger.com
(C) 2008 S. Karger AG, Basel

$1660-2110 / 09 / 1111-0039 \$ 26.00 / 0$

Accessible online at:

www.karger.com/nec
Katherine Barraclough

Department of Nephrology, St. Paul's Hospital

1081 Burrard Street, Providence Building, 6th Floor, Room 6010A

Vancouver, B.C. V6Z 1 Y6 (Canada)

Tel. +1 604682 2344, Fax +1 604806 8120, E-Mail arbieb@hotmail.com 
also has high exposure to other medications such as antiinfective and chemotherapeutic agents because of increased susceptibility to opportunistic illness as a consequence of immune deficiency. To properly predict the effects of parent drugs and metabolites and to properly adjust doses so as to ensure drug efficacy and safety, accurate assessment of kidney function is required.

Further, kidney disease is common with HIV infection, with an increasing number of different forms of kidney disease reported in this population [2]. The presence of proteinuria and/or decreased renal function in the HIV population has been associated with increased mortality and worse outcomes [3]. In order to appropriately identify, manage and prognosticate in these patients, accurate assessment of kidney function is essential.

Kidney function is commonly assessed by the use of serum creatinine concentration ( $\mathrm{SCr}$ ), creatinine clearance $(\mathrm{CrCl})$ and glomerular filtration rate (GFR) estimation based on estimation equations. Creatinine generation varies according to age, gender, race and body size because of differential muscle mass and metabolism [4]. Creatinine also undergoes proximal tubular and extrarenal clearance, making it imperfect for GFR measurement. Direct measurement of $\mathrm{CrCl}$ with timed (24-hour) urine collections is also unreliable, largely due to collection inaccuracies [4].

Consequently, estimation equations are recommended as the preferred method for assessment of GFR [4]. However, the commonly used Modification of Diet in Renal Disease (MDRD) [5] and Cockcroft-Gault (CG) [6] equations have several limitations, one of which relates to their derivation and validation in specific populations. While there has been extensive evaluation of these equations in other populations, performance has been variable. Estimation equations based on cystatin $\mathrm{C}$ have been proposed as a promising alternative [7].

There has been no validation of GFR estimation equations in the HIV population. There have been concerns that they may be less valid. Firstly, it is known that estimation equations are less reliable in patients with extremes of muscle mass, relevant to the often malnourished HIV population. We previously described a case of severe acute kidney injury in an emaciated HIV-infected man that went unrecognized because of reliance on estimated GFR [8]. The entity of HIV-associated lipodystrophy has also emerged, which includes fat atrophy, accumulation or redistribution without substantial loss of lean tissue mass [9]. The effect of such abnormal body composition on estimation equations is unclear. Secondly, while HAART has revolutionized HIV treatment, it involves complex regimens accompanied by a vast array of toxicities, including renal tubular toxicities [10]. There are now 22 FDA-approved antiretroviral drugs, with additional investigational agents in testing. Many have uncertain side effect profiles, which may include interference with creatinine generation or excretion. Thirdly, HIV is known to infect and disrupt the glomerular epithelium, with the potential development of HIV-associated nephropathy [11]. HIV can also infect mesangial and tubular cells and can persist in renal cells as a viral reservoir even after effective therapy [12]. It is not known whether the HIV virus itself may influence any creatinine-based GFR estimation.

In this pilot study, we compare all of the currently available methods of kidney function assessment with 99m Tc-diethylenetriaminepentaacetic acid (Tc-99m Pentetate) GFR measurements in HIV-infected adults across a range of SCr values. Our purpose was to explore whether the atypical characteristics of this population impacted on GFR estimation.

\section{Patients and Methods}

\section{Patients}

HIV-infected adults over 18 years of age followed in the HIV clinic at St. Paul's Hospital, Vancouver, B.C., Canada, were eligible for inclusion in the study. Exclusion criteria included acute illness, unstable renal function [variation in SCr of $>27 \mu \mathrm{mol} / 1$ $(0.3 \mathrm{mg} / \mathrm{dl})$ in the previous 6 weeks or since last measurement], pregnancy or a plan to become pregnant and refusal to provide informed consent. Consecutive patients from the existing HIV clinic population fulfilling the eligibility criteria were approached. A convenience sample of 27 patients was included.

The University of British Columbia/Providence Health Care Research Ethics Board approved the protocol. All patients provided written informed consent. Given the exploratory nature of the study, no results were formally reported to the patients' attending physicians.

Clinical Data and Anthropometric Measurements

Medical records were reviewed to obtain demographic data, information on the presence of comorbidities and medications. Data were confirmed with the patients. The patients' blood pressure, height and weight were recorded, and their body mass index (BMI) was calculated. Ideal body weight (IBW) was also calculated using the Devine formulae:

males: IBW $(\mathrm{kg})=50+0.9 \mathrm{~kg} / \mathrm{each} \mathrm{cm}$ over $152 \mathrm{~cm}$

females: IBW $(\mathrm{kg})=45.5+0.9 \mathrm{~kg} /$ each $\mathrm{cm}$ over $152 \mathrm{~cm}$.

Because of imperfect correlation between BMI and body composition [13], mid-upper arm circumference (AC) and triceps skinfold thickness (TSF) were also measured, being the anthropometric parameters most widely used to assess the composition of total body weight [14]. Mid-upper arm fat area (AFA) and arm 
muscle area (AMA) were calculated based on measurements of AC and TSF, according to the following formulae [14]:

total upper arm area $\left(\mathrm{cm}^{2}\right)=\mathrm{AC}^{2} / 4 \times \pi$

uncorrected AMA $\left(\mathrm{cm}^{2}\right)=\left[\mathrm{AC}-(\mathrm{TSF} \times \pi)^{2}\right] / 4 \times \pi$ corrected AMA $\left(\mathrm{cm}^{2}\right)$ :

males: corrected AMA = uncorrected AMA $-10 \mathrm{~cm}^{2}$

females: corrected AMA - uncorrected AMA - $6.5 \mathrm{~cm}^{2}$

AFA $\left(\mathrm{cm}^{2}\right)=$ total upper arm area - AMA.

Values were compared with reference data from National Health and Nutrition Examination Surveys I and II and classified according to the categories derived statistically from these data [14].

\section{GFR Measurement}

GFR was determined from the renal clearance of Tc-99m Pentetate. Patients received $500 \mathrm{ml}$ of intravenous saline prior to intravenous injection of $18.5 \mathrm{MBq}$ of Tc-99m Pentetate. Blood was sampled at 60 and $180 \mathrm{~min}$. The average of the 2 GFR measurements was calculated and standardized for a body surface area (BSA) of $1.73 \mathrm{~m}^{2}$. The coefficient of variation of renal clearance of Tc-99m Pentetate was 3\%. Nuclear renograms were read by a radiologist blinded to the laboratory results and clinical status of the patients.

\section{Creatinine Assay}

Blood samples were obtained immediately prior to nuclear GFR. All creatinine measurements were performed at the same laboratory, where an enzymatic colorimetric assay (standardized against isotope dilution mass spectrometry) was used, with a Roche/Hitachi analyzer. The laboratory reported a coefficient of variation of $0.9 \%$ for the assay during the period of study.

Creatinine-Based Estimation of GFR

We used 2 formulae to estimate GFR from SCr, i.e. the 4-variable MDRD formula and the CG formula.

(1) The 4-variable MDRD formula [15]:

$\operatorname{MDRD}-G F R\left(\mathrm{ml} / \mathrm{min} / 1.73 \mathrm{~m}^{2}\right)=30,849 \times$

$[\mathrm{SCr}(\mu \mathrm{mol} / \mathrm{l})]^{-1.154} \times[\text { age }(\text { years })]^{-0.203}[\times 0.742$ if female $]$.

The original equation [5] was re-expressed in this form in 2005 for use with a standardized SCr assay, which yields 5\% lower values for the SCr concentration. No adjustment was made for race, as there were no African-Americans included in the current study.

(2) The Cockcroft and Gault formula [6]:

$\mathrm{CG}-\mathrm{CrCl}(\mathrm{ml} / \mathrm{min})=[140-$ age $($ years $) \times$ weight $(\mathrm{kg})] / 0.814$ $\times \operatorname{SCr}(\mu \mathrm{mol} / \mathrm{l})[\times 0.85$ if female $]$.

CG-CrCl estimations using actual body weight were also standardized for IBW and for estimated BSA. The BSA correction was performed using the Mosteller formula [16]:

$$
\text { BSA }\left(\mathrm{m}^{2}\right)=([\text { height }(\mathrm{cm}) \times \text { weight }(\mathrm{kg})] / 3,600)^{0.5} \text {. }
$$

\section{Cystatin C}

Serum for cystatin C levels was collected at the time of SCr collection. Cystatin $\mathrm{C}$ was measured by quantitative sandwich enzyme immunoassay (R\&D Systems ELISA), standardized against a highly purified NSO-expressed recombinant human cystatin C.
The within-assay and between-assay coefficients of variation for the assay were $3.1-6.6 \%$ and 5-7\%, respectively. GFR was estimated from the cystatin $\mathrm{C}$ measurement using the following equation [17]:

$$
\text { cystatin C GFR }=86.7 / \text { cystatin }-4.2 \text {. }
$$

\section{4-Hour Urine Collection}

Urine was collected over a 24-hour period that included the day on which Tc-99m Pentetate-GFR was measured. Patients were given verbal and written instructions regarding appropriate collection technique, then advised to collect urine at home as usually occurs in clinical practice. $\mathrm{CrCl}$ was calculated using the following equation:

$$
\mathrm{CrCl}=\mathrm{UCr} / \mathrm{SCr} \times \mathrm{Qu}
$$

where UCr and SCr were the urine and serum concentrations of creatinine, respectively, and Qu was the urine volume divided by the time of the actual collection. $\mathrm{CrCl}$ estimates were adjusted for BSA.

\section{Statistical Analysis}

The baseline characteristics of the study cohort were summarized according to the level of nuclear GFR, i.e. $<60,60-90$ and $>90 \mathrm{ml} / \mathrm{min} / 1.73 \mathrm{~m}^{2}$. Descriptive statistics used for continuous and categorical variables were medians with interquartile range (IQR) and percentages, respectively, unless otherwise indicated.

We assessed the performance of 6 GFR estimates in a similar manner to that described by Ibrahim et al. [18]; specifically, the 4 assessment measures used were:

(1) Bias: difference between estimated GFR and nuclear GFR, i.e. (estimated GFR - nuclear GFR); also known as prediction error.

(2) Precision: the value of $\mathrm{R}^{2}$ from simple linear regression with nuclear GFR as the outcome and estimated GFR as the predictor. $\mathrm{R}^{2}$ represents the percentage of variability in nuclear GFR that can be explained by the estimated GFR.

(3) Accuracy: absolute value of the bias expressed as a percentage of the nuclear GFR, i.e. absolute GFR (estimated GFR - nuclear GFR)/nuclear GFR $\times 100 \%$.

(4) Relative accuracy: the percentage of estimated GFR falling within 10,20, 30 and $50 \%$ of the nuclear GFR.

Results of the performance assessment were summarized in a single table. The root mean square error (MSE), which assesses the quality of the estimated GFR in terms of its variation and unbiasedness, was also provided. Bland-Altman plots for the 6 GFR estimates were also generated. Analyses were carried out using SAS software, version 9.1 (SAS Institute, Cary, N.C., USA); graphs were completed using statistical software S-PLUS, version 7.0 (Insightful Corporation).

\section{Results}

\section{Clinical Characteristics}

Table 1 shows the baseline demographic and clinical characteristics of the cohort according to measured GFR. Most were male and Caucasian, with a mean age of 52 
Table 1. Summary of baseline characteristics

\begin{tabular}{|c|c|c|c|c|}
\hline \multirow[t]{2}{*}{ Variable } & \multirow[t]{2}{*}{ Total } & \multicolumn{3}{|c|}{$\mathrm{GFR}, \mathrm{ml} / \mathrm{min} / 1.73 \mathrm{~m}^{2}$} \\
\hline & & $<60$ & $60-90$ & $>90$ \\
\hline Patients & 27 & 3 & 10 & 14 \\
\hline Serum creatinine, $\mu \mathrm{mol} / \mathrm{l}$ & $89(76,95)$ & $147(116,177)$ & $89(80,92)$ & $78(64,90)$ \\
\hline Tc- $99 \mathrm{~m}$ Pentetate-GFR, $\mathrm{ml} / \mathrm{min} / 1.73 \mathrm{~m}^{2}$ & $91(84,114)$ & $52(33,52)$ & $86(78,87)$ & $112(93,135)$ \\
\hline Age, years & $52 \pm 9$ & $62 \pm 7$ & $52 \pm 8$ & $49 \pm 9$ \\
\hline Male, n (\%) & $23(85)$ & $2(67)$ & $9(90)$ & $12(86)$ \\
\hline Actual body weight, $\mathrm{kg}$ & $70(62,80)$ & $61(60,67)$ & $66(59,78)$ & $75(68,83)$ \\
\hline IBW, kg & $68(61,75)$ & $62(57,71)$ & $68(62,73)$ & $70(57,78)$ \\
\hline $\mathrm{BSA}, \mathrm{m}^{2}$ & $1.83(1.73,1.97)$ & $1.73(1.67,1.73)$ & $1.77(1.63,1.96)$ & $1.90(1.79,2.00)$ \\
\hline BMI & $24.6(21.7,26.0)$ & $22.5(18.5,26.2)$ & $23.3(21.1,25.0)$ & $25.0(22.0,26.8)$ \\
\hline \multicolumn{5}{|l|}{ Arm muscle area, \% } \\
\hline Muscle deficit & 44 & 33 & 70 & 29 \\
\hline Below average & 30 & 67 & 20 & 29 \\
\hline Average & 19 & 0 & 10 & 29 \\
\hline Above average & 7 & 0 & 0 & 13 \\
\hline \multicolumn{5}{|l|}{ Arm fat area, $\%$} \\
\hline Fat deficit & 37 & 67 & 40 & 29 \\
\hline Below average & 11 & 0 & 10 & 14 \\
\hline Average & 37 & 33 & 40 & 36 \\
\hline Fat excess & 15 & 0 & 10 & 21 \\
\hline Diabetes, $\%$ & 7 & 67 & 0 & 0 \\
\hline Hypertension, \% & 26 & 67 & 30 & 14 \\
\hline CD 4 count, cells $/ \mu l$ & $290(200,460)$ & $230(80,460)$ & $230(200,290)$ & $360(280,470)$ \\
\hline HIV-1 viral load $<50$ copies $/ \mathrm{ml}, \%$ & 70 & 67 & 60 & 79 \\
\hline Albumin, $g / 1$ & $44(43,46)$ & $45(42,49)$ & $44(43,46)$ & $44(43,46)$ \\
\hline Prealbumin, mg/l & $273(248,312)$ & $346(312,490)$ & $253(198,310)$ & $273(256,304)$ \\
\hline Total cholesterol, mmol/l & $4.2(3.9,5.0)$ & $6.4(3.6,8.4)$ & $3.9(3.9,5.0)$ & $4.3(4.0,4.6)$ \\
\hline $\mathrm{HDL}-\mathrm{C}, \mathrm{mmol} / \mathrm{l}$ & $1.1(0.9,1.7)$ & $1.2(0.9,1.7)$ & $1.4(1.1,1.8)$ & $1.0(0.9,1.6)$ \\
\hline LDL-C, $\mathrm{mmol} / \mathrm{l}$ & $2.1(1.5,3.1)$ & $3.3(1.4,5.5)$ & $2.4(1.7,3.1)$ & $2.0(1.5,2.8)$ \\
\hline Triglycerides, mmol/l & $1.8(1.0,2.9)$ & $3.9(1.0,7.5)$ & $1.4(1.0,1.8)$ & $1.8(1.0,2.6)$ \\
\hline Hematuria, \% & 11 & 0 & 10 & 14 \\
\hline Proteinuria, $\%$ & 44 & 67 & 50 & 36 \\
\hline Albumin/creatinine ratio & $2(0.7,8.8)$ & $8.8(1.1,15.3)$ & $2.1(0.9,6.6)$ & $1.0(0.7,5.0)$ \\
\hline Total protein excretion, g/day & $0.23(0.10,0.63)$ & $0.32(0.07,0.63)$ & $0.50(0.14,1.60)$ & $0.15(0.10,0.38)$ \\
\hline Current ARV medications, number/patient & $5(4,6)$ & $5(4,6)$ & $5(4,5)$ & $4.5(4,6)$ \\
\hline \multicolumn{5}{|l|}{ ARV class, $\%$} \\
\hline NNRTIs & 22 & 0 & 40 & 14 \\
\hline NRTIs & 100 & 100 & 100 & 100 \\
\hline Protease inhibitors & 96 & 100 & 90 & 100 \\
\hline Fusion inhibitors & 15 & 0 & 30 & 7 \\
\hline Integrase inhibitors & 11 & 33 & 0 & 14 \\
\hline
\end{tabular}

Values expressed are medians (IQR), except for mean (SD) for age. HDL-C = High-density lipoprotein cholesterol; LDL-C = lowdensity lipoprotein cholesterol; ARV = antiretroviral; NNRTI = nonnucleoside reverse transcriptase inhibitor; NRTI = nucleoside reverse transciptase inhibitor.

years. The median (IQR) SCr level for the cohort was 89 $(76,95) \mu \mathrm{mol} / \mathrm{l}[1(0.9,1.1) \mathrm{mg} / \mathrm{dl}]$. The median (IQR) Tc$99 \mathrm{~m}$ Pentetate-GFR was $91(84,114) \mathrm{ml} / \mathrm{min} / 1.73 \mathrm{~m}^{2}$. Fifty-six percent of patients (15/27) had a nuclear GFR in the normal range. Sixteen patients (59\%) had a normal BMI.
Despite only 1 patient (4\%) having a BMI in the underweight range and only 5 patients (19\%) having abnormal prealbumin and albumin levels, 37\% (10/27) and 44\% $(12 / 27)$ of patients were classified as having a deficit of fat or muscle, respectively. Only a minority of patients was 
Table 2. Performance of prediction methods in predicting nuclear GFR

\begin{tabular}{|c|c|c|c|c|c|c|}
\hline & \multicolumn{6}{|c|}{ Estimated GFR } \\
\hline & MDRD & CG ABW & CG IBW & CG BSA & 24-hour $\mathrm{CrCl}$ & cystatin C GFR \\
\hline Patients & 27 & 27 & 27 & 27 & 25 & 27 \\
\hline Bias, $\mathrm{ml} / \mathrm{min}$ per $1.73 \mathrm{~m}^{2}$ & $-10(-21,2)$ & $-4(-14,10)$ & $-8(-26,10)$ & $7(-15,20)$ & $6(-12,13)$ & $-29(-53,-16)$ \\
\hline Precision, $\mathrm{R}^{2}$ & 0.55 & 0.34 & 0.40 & 0.21 & 0.60 & 0.18 \\
\hline$(95 \% \mathrm{CI})$ & $(0.33,0.77)$ & $(0.08,0.60)$ & $(0.15,0.65)$ & $(0.00,0.45)$ & $(0.39,0.81)$ & $(0.00,0.41)$ \\
\hline Root MSE & 17.9 & 21.8 & 20.8 & 23.8 & 17.5 & 24.3 \\
\hline Accuracy, \% & $18(10,25)$ & $16(8,38)$ & $19(8,33)$ & $21(10,42)$ & $14(9,22)$ & $32(20,49)$ \\
\hline \multicolumn{7}{|l|}{ Relative accuracy, n } \\
\hline $10 \%$ & $6(22)$ & $9(33)$ & $8(30)$ & $7(26)$ & $7(28)$ & $3(11)$ \\
\hline $20 \%$ & $16(59)$ & $15(56)$ & $15(56)$ & $13(48)$ & $17(68)$ & $6(22)$ \\
\hline $30 \%$ & $24(89)$ & $19(70)$ & $20(74)$ & $19(70)$ & $22(88)$ & $11(41)$ \\
\hline $50 \%$ & 27 (100) & $23(85)$ & $26(96)$ & $22(81)$ & $24(96)$ & $22(81)$ \\
\hline
\end{tabular}

Values expressed are medians (IQR) or numbers (percentages). The 95\% CIs for precision are also provided. ABW = Actual body weight.

hypertensive or diabetic $[26 \%(7 / 27)$ and $7 \%(2 / 27)$, respectively]. At least one abnormality in the lipid profile was present in $52 \%$ of patients (14/27). The median (IQR) CD4 count was $290(200,460)$ cells $/ \mathrm{mm}^{3}$. Only 5 patients (19\%) had a CD 4 count of $<200$ cells $/ \mathrm{mm}^{3}$, and most patients [70\% (19/27)] had suppressed viral load ( $<50$ copies $/ \mathrm{ml}$ ). All patients were receiving HAART; $100 \%$ were being treated with nucleoside reverse transcriptase inhibitors [including tenofovir in 17/27 (63\%)], 22\% with nonnucleoside reverse transcriptase inhibitors, $96 \%$ with protease inhibitors, $15 \%$ with a fusion inhibitor and $11 \%$ with an integrase inhibitor. Forty-one percent of patients were receiving 3 or more classes of drugs, and the median (IQR) number of drugs per patient was $5(4,6)$. No patients were receiving trimethoprim-sulfamethoxazole for Pneumocystis jiroveci prophylaxis (2 patients were receiving dapsone therapy) or any other medication known to impact on creatinine excretion.

\section{Comparisons of the Different Methods of GFR \\ Estimation}

A comparison of the performance of the various methods of GFR estimation is shown in table 2. Figure 1 displays the scatter plots between various GFR estimates (horizontal axis) and their corresponding Tc-99m Pentetate-GFR. The regression line given in each plot estimates the true relationship between the GFR estimate and nuclear GFR; the corresponding $95 \%$ confidence interval for the regression line reflects the confidence boundary of where the true relationship may lie. The bias and relative accuracy of the various estimation methods are depicted in figures 2 and 3 , respectively, using Bland-Altman plots.

Abbreviated MDRD Estimate. The MDRD equation showed slightly greater bias than all other creatininebased methods of kidney function assessment (fig. 1,2). The median accuracy of the MDRD formula and CG$\mathrm{CrCl}$ using actual body weight were similar (18 vs. $16 \%$, respectively). However, the MDRD formula showed greater precision and a smaller root MSE than the CG formulae, and relative accuracy within $30 \%$ of nuclear GFR was greater for the MDRD formula than for all other methods of kidney function assessment. Figure 3 shows that all except 3 values (11\%) fell within 30\% of nuclear GFR. Figures 2 and 3 also demonstrate that bias was greater and accuracy was lower at higher levels of GFR.

CG Estimates. CG-CrCl (using actual body weight) showed minimal bias but significant imprecision and a larger root MSE. Relative accuracy was lower than for the MDRD formula, with 33 and $70 \%$ of estimates falling within 10 and $30 \%$ of nuclear GFR, respectively. Adjustment for IBW and BSA increased bias; adjustment for IBW had little effect on accuracy but slightly increased precision, while adjustment for BSA decreased both precision and accuracy. All CG plots showed high variability, with the appearance of random scatter across all ranges of nuclear GFR.

24-Hour Urine $\mathrm{CrCl}$. The median (IQR) bias of 24hour $\mathrm{CrCl}$ was $6(-12,13) \mathrm{ml} / \mathrm{min} / 1.73 \mathrm{~m}^{2}$, which was lower (in absolute magnitude) than that seen with the MDRD formula $\left[-10(-21,2) \mathrm{ml} / \mathrm{min} / 1.73 \mathrm{~m}^{2}\right]$. As shown 


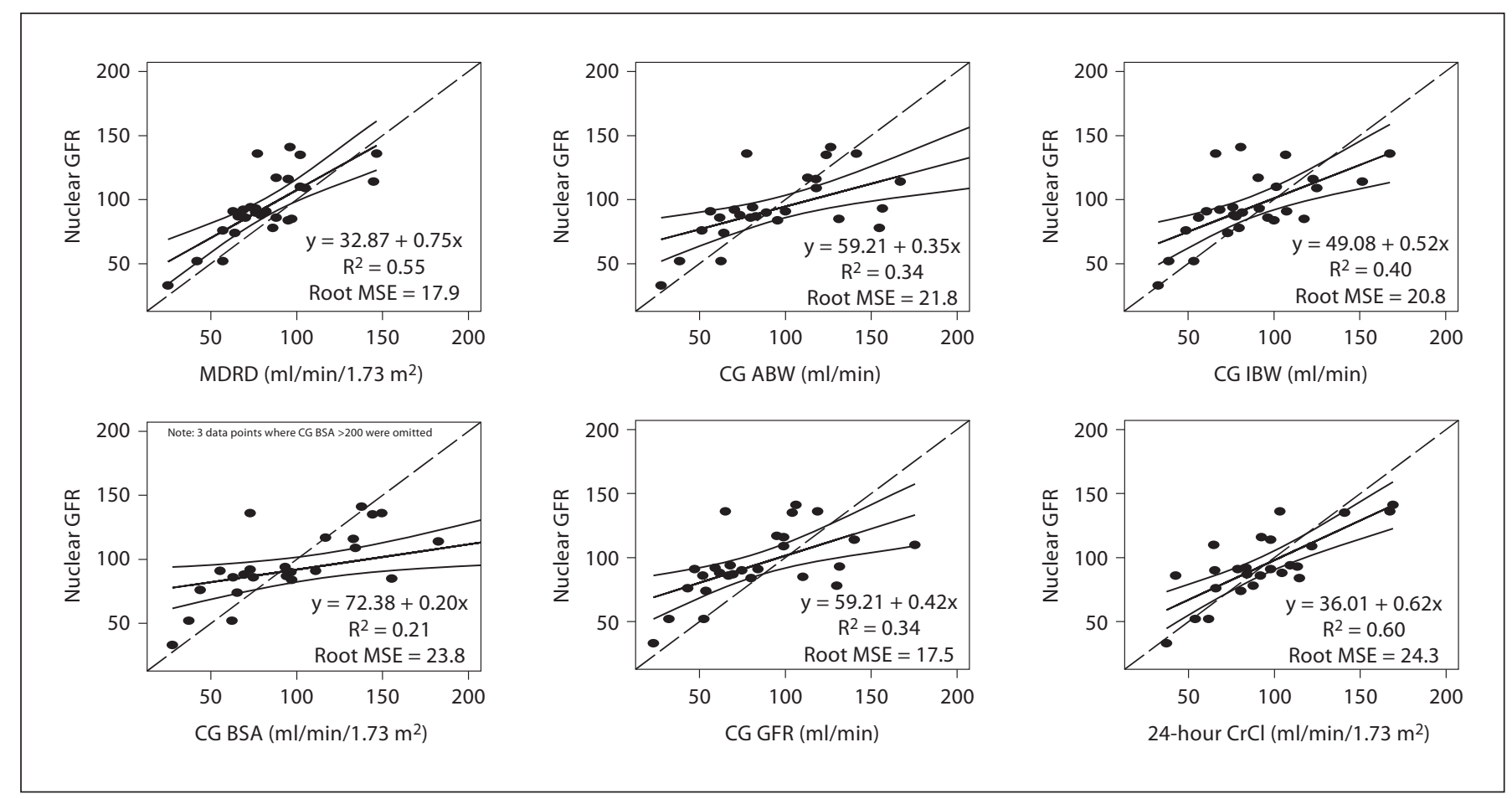

Fig. 1. Relationship between nuclear GFR (y-axis) and the various GFR or CrCl estimates (x-axis). The diagonal straight lines represent the regression lines. The curved lines represent the $95 \%$ confidence intervals for the regression lines. ABW = Actual body weight.

in figure 2, there was no consistent pattern to the direction of bias, with $\mathrm{CrCl}$ measurements both over- and underestimating nuclear GFR. Twenty-four-hour $\mathrm{CrCl}$ showed the greatest precision and accuracy and had the smallest root MSE of all estimation methods in our data. Relative accuracy was similar to that seen with the MDRD formula; the results were within 10 and $30 \%$ of the nuclear GFR in 28 and $88 \%$, respectively (fig. 3). As with the MDRD formula, bias was greater and accuracy was lower at higher levels of GFR (fig. 2, 3). However, this increased error was apparent at lower levels of GFR for 24hour $\mathrm{CrCl}$ (around $80-110 \mathrm{ml} / \mathrm{min} / 1.73 \mathrm{~m}^{2}$ ) than for the MDRD formula (around $120 \mathrm{ml} / \mathrm{min} / 1.73 \mathrm{~m}^{2}$ ).

Cystatin C GFR Estimate. Cystatin C showed the greatest bias of all methods, with most estimates underestimating GFR. It was also the least precise and accurate of methods, and had the largest root MSE. Only $41 \%$ of estimates fell within $30 \%$ of nuclear GFR.

\section{Discussion}

Two previous studies have compared GFR estimation equations with measured $\mathrm{CrCl}$ by 24 -hour urine collection in HIV patients $[19,20]$. Conducted in the preHAART era, it is interesting to note the differences in the HIV populations examined by these studies; no patients were receiving antiretroviral therapy, most were markedly immunosuppressed with acquired immune deficiency syndrome-related illnesses and the majority of patients were underweight (IBW greater than actual body weight).

This study is the first to compare the various methods of GFR estimation against nuclear renogram in HIV-infected patients receiving treatment in the current era. It evaluates the performance of all the currently available methods of kidney function estimation in $27 \mathrm{HIV}$-infected patients across a range of SCr values. All patients were receiving antiretroviral therapy and the majority had well-controlled HIV disease; a minority were malnourished, but many had abnormal body composition. We demonstrate that the performance of the abbreviated MDRD equation is superior to GFR estimation by the CG 


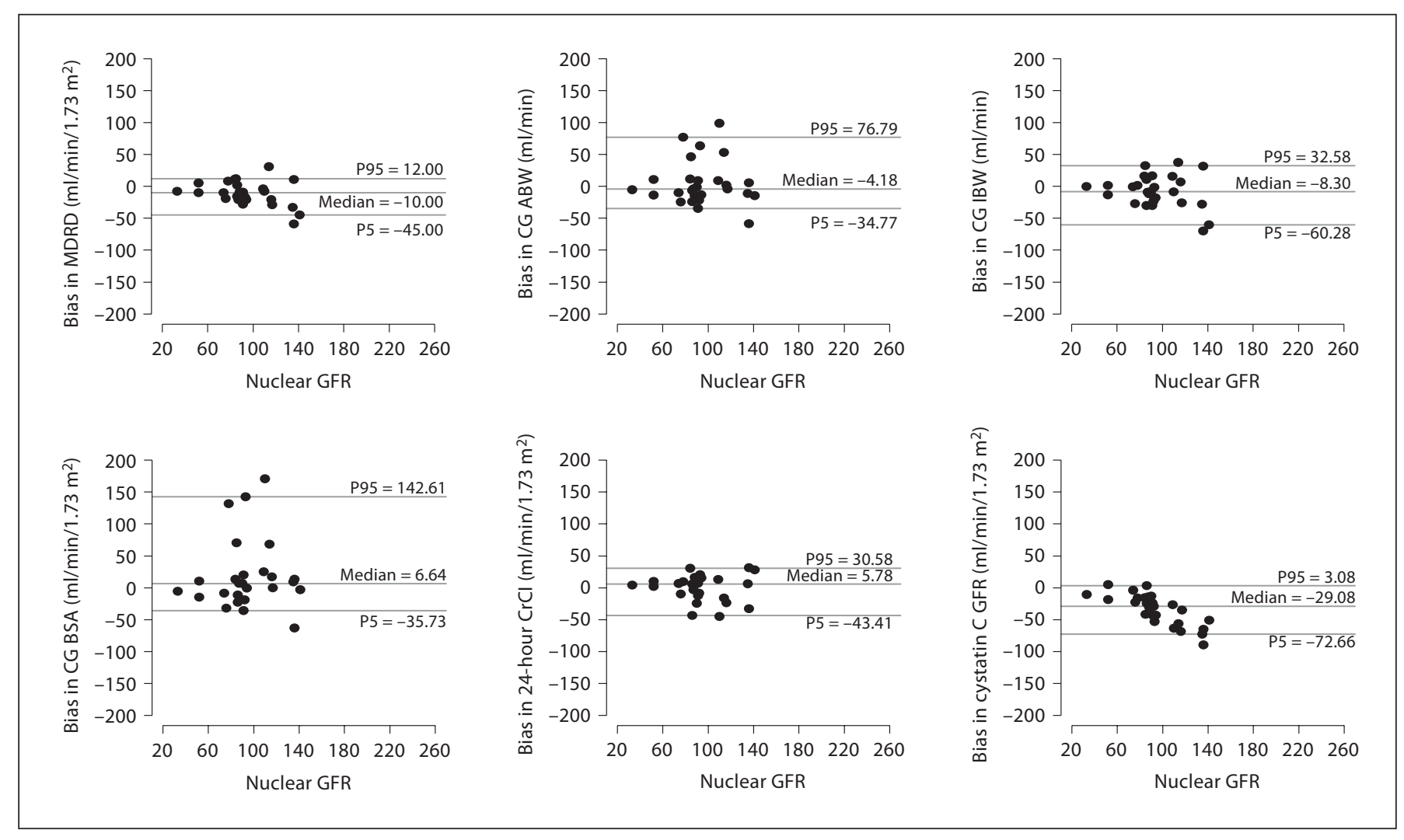

Fig. 2. Bland-Altman plots displaying the bias of the various GFR or $\mathrm{CrCl}$ estimates (y-axis) across all ranges of nuclear GFR (x-axis). The median bias (and 5th and 95th percentiles) are represented by the horizontal lines. $\mathrm{ABW}=$ Actual body weight.

formulae, with an accuracy that is similar to that seen in previous validation studies involving patients with similar kidney function [21-23]. We also show that the performance of $\mathrm{CrCl}$ measurement by 24 -hour urine collection is similar to that of the MDRD formula for those patients with GFR less than $90 \mathrm{ml} / \mathrm{min} / 1.73 \mathrm{~m}^{2}$, although it performs less well at higher levels of GFR. Cystatin C GFR estimation was inferior to all of the creatinine-based methods. The acceptable performance of the MDRD formula and 24-hour urine $\mathrm{CrCl}$ measurement suggests that both methods are suitable for GFR estimation in the HIVinfected population with clinical characteristics as described. Although larger validation studies are needed, these findings alleviate some of the concern regarding use of these tests in this unique population, particularly in relation to the effects of HAART and abnormal body composition on the estimation of kidney function.

The MDRD study equation was derived from data from primarily white subjects with nondiabetic kidney disease and a mean GFR of $40 \mathrm{ml} / \mathrm{min} / 1.73 \mathrm{~m}^{2}$ [5]. In this population, the MDRD equation gave a median percentage absolute error of $11.5 \%$. Large validation studies have tested the equation in alternative populations. It has been shown to be less accurate in healthy subjects without kidney disease [21,23], those $<18$ or $>70$ years, Asians, those with unusual body mass, pregnant subjects and possibly transplant recipients $[22,24-26]$. We found that the accuracy of the MDRD formula in our HIV study population (18\%) was lower than that observed in the MDRD study. This likely reflects the generally well-preserved kidney function in our study group. Consistent with this interpretation, the Bland-Altman plots (fig. 2, 3) demonstrate an increase in bias and reduction in accuracy at higher levels of GFR. Compared to the other methods, we found that the MDRD formula showed slightly greater bias but was more precise than the CG formulae, and performed better than all other methods of kidney function assessment with respect to relative accuracy within $30 \%$ of nuclear GFR. This is considered to be a level of accuracy sufficient for clinical decision making [4]. This rela- 


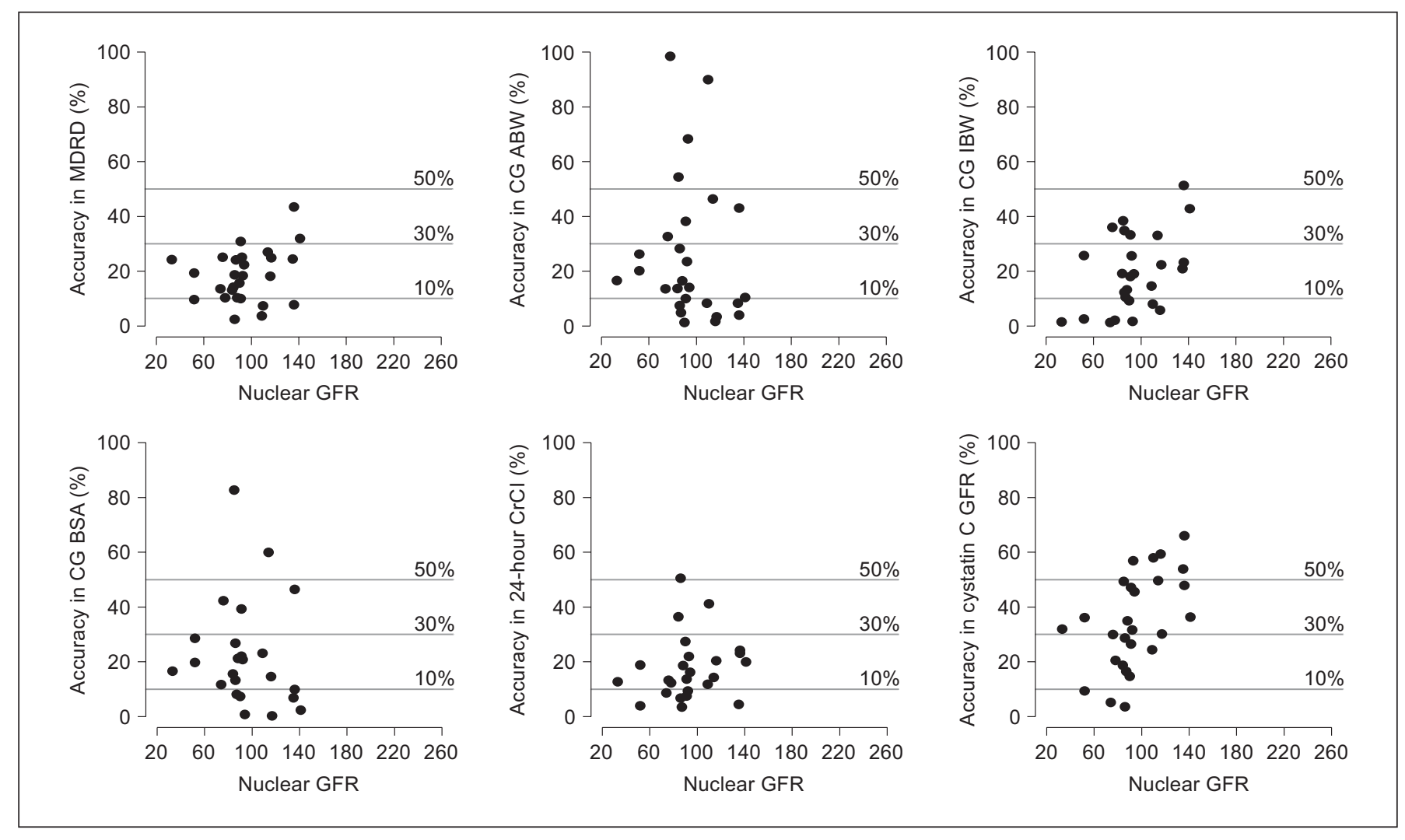

Fig. 3. Scatter plots between accuracy (or percentage absolute bias) of the various GFR or CrCl estimates (y-axis) and nuclear GFR (x-axis). Accuracy within 10, 30 and 50\% of nuclear GFR are represented by the horizontal lines.

tive accuracy was similar in our study population to that observed in the MDRD study.

The CG formula was developed in a Caucasian, predominantly male population, with no information about disease status [6]. It was designed to predict $\mathrm{CrCl}$ rather than GFR. It is not surprising, then, that this equation has performed worse than the MDRD formula in most comparative studies. The relative bias in estimating GFR using CG has varied markedly across studies (from -14 to $+25 \%$ ) [4]. We found that the traditional CG equation, incorporating actual body weight and age, showed less bias $(-4 \mathrm{ml} / \mathrm{min})$ than all other methods. However, it was also less precise than other methods, and a substantially lower proportion of values fell within $30 \%$ of nuclear GFR. Given the potential abnormalities of body weight composition that may exist with HIV, we also estimated CG-CrCl using IBW. This led to marginally improved precision and relative accuracy, but performance was still inferior to that of MDRD. Standardizing results for BSA also failed to improve performance.
Measuring 24-hour $\mathrm{CrCl}$ to assess GFR has consistently been shown to be no more reliable than estimating GFR from prediction equations [4]. In addition to collection errors, this can be attributed to diurnal variation in GFR and day-to-day variation in creatinine excretion [4]. Further, as with CG, urine collection measures $\mathrm{CrCl}$ rather than GFR. In keeping with the norm, 2 of our urine collections were incomplete and were therefore considered inadequate for analysis by the laboratory. For the remaining collections, we found performance to be similar to that of the MDRD formula for those patients with GFR less than $90 \mathrm{ml} / \mathrm{min} / 1.73 \mathrm{~m}^{2}$, but inferior at higher levels of GFR. Thus, while overall precision and accuracy are reasonable, the lack of clear superiority, the inconvenience and the collection inaccuracies associated with this measurement make the MDRD method preferable for routine use.

A criticism of all currently available methods of GFR estimation relates to their reliance on SCr. Cystatin C is an alternative endogenous filtration marker that is pro- 
duced by all nucleated cells at a constant rate. The advantage of cystatin $\mathrm{C}$ includes the fact that reference ranges do not depend on sex, muscle mass or age $[7,27]$. Most studies suggest at least equivalence of cystatin $\mathrm{C}$ with creatinine-based GFR estimations, with possible superiority in those patients with abnormal body mass and higher levels of GFR $[7,28]$. One previous study that examined cystatin C levels in HIV-infected adults demonstrated GFR underestimation by cystatin $\mathrm{C}$ when compared to the MDRD formula. Cystatin C levels correlated positively with HIV RNA and negatively with the CD4+ cell count [29], suggesting an interaction between HIV infection and cystatin C levels. We also found that cystatin C underestimated nuclear GFR. The performance of the cystatin C GFR equation was inferior to all of the creatinine-based methods, with less than half of estimates falling within $30 \%$ of nuclear GFR. One possible explanation lies in the estimation equation selected (derived using a similar gold-standard GFR measurement but in a diabetic cohort). However, we found no improvement in performance with the use of alternative equations (data not shown). Other potential explanations include inferiority of the enzymatic immunoassay method of detecting cystatin $\mathrm{C}$ or a unique characteristic of an HIV cohort.

The primary limitation of this study is the small sample size. Nevertheless, our results are concordant with those of previous studies. A second limitation is that our study participants were a referred, predominantly Caucasian population with generally well-controlled HIV and metabolic parameters. Thus, our population is representative of treated HIV patients in the HAART era, but our results may not be generalizable to the HIV community at large, particularly those from developing countries with more profound immunodeficiency or AIDSassociated wasting. Women were also underrepresented, limiting the application of our results to this group.

A further possible limitation relates to the use of Tc$99 \mathrm{~m}$ Pentetate as a filtration marker. While high correlation of Tc-99m Pentetate-GFR to simultaneous measures of inulin clearance has been reported [30], other studies have questioned the performance of this filtration marker [21,31]. Regardless of the filtration marker used, a measurement error of 5-20\% has been reported with the use of exogenous markers to measure GFR [32]. Causes for this intratest variability have not been well studied [33], but may stem from short-term biological variation in GFR. We performed nuclear measurements as close together with blood and urine measurements as practically possible to allow for accurate comparison between methods. To account for diurnal GFR variation [33], we performed all nuclear measurements at the same time of day. Regardless, Tc-99m Pentetate-GFR reflects GFR over $4 \mathrm{~h}$, whereas SCr reflects the average GFR over a much longer period. Thus, it is possible that this 'gold standard' test of kidney function may not be truly reflective. Further, hour-to-hour variation can occur due to changes in renal blood flow, and also with meals due to protein- and salt-induced hyperfiltration [33]. As we did not standardize diet on the day of testing, this may have led to misclassification of GFR status (i.e. $>90$ vs. $60-90 \mathrm{ml} / \mathrm{min} /$ $1.73 \mathrm{~m}^{2}$ ).

Despite these limitations, this is the first study to evaluate the validity of the various methods of estimation of kidney function against nuclear renogram in the HIVinfected population. We describe a well-characterized cohort of HIV-infected patients with generally preserved, though variable, renal function. Despite most patients having normal nutritional status and BMIs, $63 \%$ did have fat or muscle deficit, suggesting abnormal body composition. All were being treated with multiple antiretroviral medications, and the majority had controlled viral replication and relative preservation of immune function. Despite the presence of these characteristics, which have been thought to impact on the use of GFR-estimating equations, we found that the abbreviated MDRD formula performed with a level of precision and accuracy sufficient for clinical decision making. Its performance was superior to estimation by the CG formulae, regardless of whether CG was corrected for IBW or BSA. We also demonstrated that $\mathrm{CrCl}$ measurement by 24 -hour urine collection is an acceptable, though problematic, alternative measure if urine collection is required for other reasons. For unclear reasons, cystatin C GFR performed poorly in our population. Our data suggest that in those patients with treated HIV, similar to those described herein, there are no HIV-specific factors that limit the applicability of the MDRD equation. Larger validation studies are needed to confirm the findings of this pilot study.

References

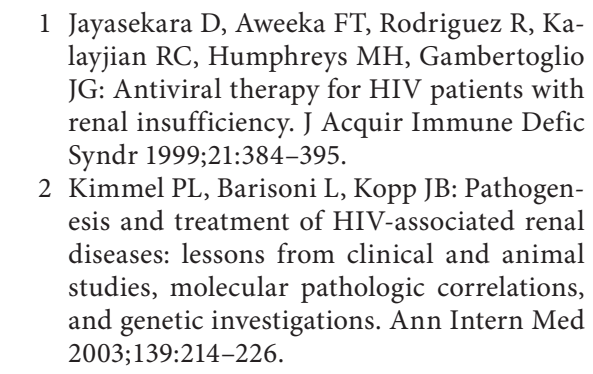

Nephron Clin Pract 2009;111:c39-c48 c47 
>3 Szczech LA, Hoover DR, Feldman JG, et al: Association between renal disease and outcomes among HIV-infected women receiving or not receiving antiretroviral therapy. Clin Infect Dis 2004;39:1199-1206.

-4 National Kidney Foundation: K/DOQI clinical practice guidelines for chronic kidney disease: evaluation, classification, and stratification. Am J Kidney Dis 2002;39:S1S266.

5 Levey AS, Bosch JP, Lewis JB, Greene T, Rogers N, Roth D: A more accurate method to estimate glomerular filtration rate from serum creatinine: a new prediction equation. Modification of Diet in Renal Disease Study Group. Ann Intern Med 1999;130:461-470.

-6 Cockcroft DW, Gault MH: Prediction of creatinine clearance from serum creatinine. Nephron 1976;16:31-41.

$\checkmark 7$ Herget-Rosenthal S, Bokenkamp A, Hofmann W: How to estimate GFR - serum creatinine, serum cystatin $\mathrm{C}$ or equations? Clin Biochem 2007;40:153-161.

8 Barraclough K, Harris M, Montessori V, Levin A: An unusual case of acute kidney injury due to vancomycin - lessons learnt from reliance on eGFR. Nephrol Dial Transplant 2007;22:2391-2394.

$\checkmark 9$ Wanke CA: Epidemiological and clinical aspects of the metabolic complications of HIV infection. The fat redistribution syndrome. AIDS 1999;13:1287-1293.

-10 Sterne JA, Hernan MA, Ledergerber B, Tilling K, Weber R, Sendi P, Rickenbach M, Robins JM, Egger M; Swiss HIV Cohort Study: Long-term effectiveness of potent antiretroviral therapy in preventing AIDS and death: a prospective cohort study. Lancet 2005;366: 378-384.

-11 Ross MJ, Klotman PE: HIV-associated nephropathy. AIDS 2004;18:1089-1099.

$\checkmark 12$ Winston JA, Bruggeman LA, Ross MD, et al: Nephropathy and establishment of a renal reservoir of HIV type 1 during primary infection. N Engl J Med 2001;344:1979-1984.

13 Prentice AM, Jebb SA: Beyond body mass index. Obes Rev 2001;2:141-147.

14 Frisancho AR: New norms of upper limb fat and muscle areas for assessment of nutritional status. Am J Clin Nutr 1981;34:25402545 .
15 Levey AS, Coresh J, Greene T, et al: Expressing the Modification of Diet in Renal Disease Study equation for estimating glomerular filtration rate with standardized serum creatinine values. Clin Chem 2007;53:766-772.

16 Mosteller RD: Simplified calculation of body-surface area. N Engl J Med 1987;317: 1098.

17 MacIsaac RJ, Tsalamandris C, Thomas MC, et al: Estimating glomerular filtration rate in diabetes: a comparison of cystatin-C- and creatinine-based methods. Diabetologia 2006;49:1686-1689.

18 Ibrahim H, Mondress M, Tello A, Fan Y, Koopmeiners J, Thomas W: An alternative formula to the Cockcroft-Gault and the Modification of Diet in Renal Diseases formulas in predicting GFR in individuals with type 1 diabetes. J Am Soc Nephrol 2005;16: 1051-1060.

19 Huang E, Hewitt RG, Shelton M, Morse GD: Comparison of measured and estimated creatinine clearance in patients with advanced HIV disease. Pharmacotherapy 1996;16: 222-229.

20 Smith BL, Sarnoski TP, Dennis S, Luke DR: Failure of predicted creatinine clearance equations in HIV-seropositive patients. Int J Clin Pharmacol Ther Toxicol 1992;30:394399.

21 Lin J, Knight EL, Hogan ML, Singh AK: A comparison of prediction equations for estimating glomerular filtration rate in adults without kidney disease. J Am Soc Nephrol 2003; 14:2573-2580.

22 Bostom AG, Kronenberg F, Ritz E: Predictive performance of renal function equations for patients with chronic kidney disease and normal serum creatinine levels. J Am Soc Nephrol 2002;13:2140-2144.

23 Poggio ED, Wang X, Greene T, Van Lente F, Hall PM: Performance of the Modification of Diet in Renal Disease and Cockcroft-Gault equations in the estimation of GFR in health and in chronic kidney disease. J Am Soc Nephrol 2005;16:459-466.

-24 Froissart M, Rossert J, Jacquot C, Paillard M, Houillier P: Predictive performance of the Modification of Diet in Renal Disease and Cockcroft-Gault equations for estimating renal function. J Am Soc Nephrol 2005;16: 763-773.
25 Gaspari F, Ferrari S, Stucchi N, et al: Performance of different prediction equations for estimating renal function in kidney transplantation. Am J Transplant 2004;4:18261835.

26 Coresh J, Astor BC, Greene T, Eknoyan G, Levey AS: Prevalence of chronic kidney disease and decreased kidney function in the adult US population: Third National Health and Nutrition Examination Survey. Am J Kidney Dis 2003;41:1-12.

-27 Deinum J, Derkx FH: Cystatin for estimation of glomerular filtration rate? Lancet 2000; 356:1624-1625.

28 Newman DJ, Thakkar H, Edwards RG, Wilkie M, White T, Grubb AO, Price CP: Serum cystatin $\mathrm{C}$ measured by automated immunoassay: a more sensitive marker of changes in GFR than serum creatinine. Kidney Int 1995;47:312-318.

29 Mauss S, Berger F, Kuschak D, et al: Cystatin $\mathrm{C}$ underestimates glomerular filtration rate in HIV-infected Individuals (abstract). Antivir Ther 2007;12(suppl 2):L59-L60.

>30 Perrone RD, Steinman TI, Beck GJ, Skibinski CI, Royal HD, Lawlor M, et al: Utility of radioisotopic filtration markers in chronic renal insufficiency: simultaneous comparison of 125I-iothalamate, 169Yb-DTPA, 99mTcDTPA, and inulin. The Modification of Diet in Renal Disease Study. Am J Kidney Dis 1990;16:224-235

-31 Mahajan S, Mukhiya GK, Singh R, et al: Assessing suitability for renal donation: can equations predicting glomerular filtration rate substitute for a reference method in the Indian population? Nephron 2005;101:c128c133.

32 Stevens LA, Coresh J, Greene T, Levey AS: Assessing kidney function - measured and estimated glomerular filtration rate. N Engl J Med 2006;356:2473-2483.

33 Levey AS, Greene T, Schluchter MD, Cleary PA, Teschan PE, Lorenz RA, Molitch ME, Mitch WE, Slebert C, Hall PM, Steffes MW: Glomerular filtration rate measurements in clinical trials. Modification of Diet in Renal Disease Study Group and the Diabetes Control and Complications Trial Research Group. J Am Soc Nephrol 1993;4:11591171. 